\title{
Barriers to Care for Persons Living With HIV Post Affordable Care Act Implementation in Northern Indiana
}

\section{Scott $A^{*}$ and Beidinger-Burnett $H$ \\ Eck Institute for Global Health, University of Notre Dame, USA}

*Corresponding author: Scott A, Eck Institute for Global Health, University of Notre Dame, USA, Tel: 574-631-9227; E-mail: Ascott12@nd.edu

\section{Research Article}

Volume 1 Issue 1

Received Date: February 16, 2017

Published Date: April 03, 2017

DOI: $10.23880 /$ phoa- 16000103

\section{Abstract}

Through the lens of the Affordable Care Act, this phenomenological study qualitatively examined barriers to care for persons living with HIV (PLWH). Study participants $(n=6)$ were PLWH who experienced at least a six month "gap in care" despite having or being eligible for insurance. The data demonstrates a minimization of traditional barriers to care and the emergence of two modern barriers. The complexity of the health insurance system and the lack of recognition of a gap in care have barred the participants from accessing consistent HIV care and as a result the virus remains transmissible for $80 \%$ of the participants. These results led researchers to recommend care coordination shift to a system where HIV is managed as a chronic disease with a focus on improving health literacy through education on viral suppression, continuity of care, and navigation of the health insurance systems.

Keywords: HIV infections; Continuity of patient care; Patient Protection and Affordable Care Act; Insurance coverage; Disease management

Abbreviations: ACA: Affordable Care Act; AMAA: AIDS Ministries AIDS Assist; ART: Antiretroviral Therapy; AIDS: Acquired Immunodeficiency Syndrome; CD4: Clusters of Differentiation 4; HIP 2.0: Healthy Indiana Plans 2.0; HIV: Human Immunodeficiency Virus; PLWH: Persons living with HIV

\section{Introduction}

Human Immunodeficiency Virus (HIV) and Acquired immunodeficiency syndrome (AIDS) remain a significant burden for Indiana. In 2013, there were 506 new cases of HIV diagnosed in Indiana which ranks it $21^{\text {st }}$ of the 50 states for new diagnoses [1]. In recent years, two remarkable changes related to HIV/AIDS and treatment has occurred. First, HIV pharmacology and treatment has improved significantly. Antiretroviral therapy (ART), while not a cure for HIV, has been shown to dramatically increase the survivability of persons living with HIV (PLWH) and significantly reduce the risk of transmission [2]. Second, the World Health Organization recently changed their HIV treatment guidelines, which state ART, should commence upon HIV diagnosis regardless of clusters of differentiation 4 (CD4) counts [3]. This new guideline removes the inconsistencies that once existed with treatment initiation. As a result of these two changes, an HIV diagnosis has shifted from a once fatal condition to a manageable chronic disease.

HIV care coordination programs in Indiana provide case management to assist PLWH to coordinate health and social services to improve overall well-being and quality of life. AIDS Ministries/AIDS Assist (AMAA) is a care coordination program in Northern Indiana and has been providing services for over 25 years. Their focus is 
to assist PLWH in initiating and maintaining HIV care [4]. Unfortunately, previous studies have identified several, persistent barriers to care including cost, social stigma, transportation, convenience, fear of abandonment, feelings of shame, and child care issues [5-12]. As a result, many PLWH experience gaps in care which AMAA describes as a period of at least six months where PLWH do not see health care professionals. This gap in care interrupts the continuity of their care yielding disruptions in treatment, health status, and/or patient/doctor relationships.

The expense of care has been identified as one of the most consistent barriers to accessing medical services for HIV $[6,13]$. One study found $38 \%$ of participants reported forgoing medical care because of the financial burden [6]. The expense of care is usually alleviated by a form of insurance. A review by Uphold and Mkanta (2005) reported, "Insurance status was found to be one of the strongest factors associated with utilization patterns" among PLWH [14]. Part of the Affordable Cart Act (ACA) of 2012, which is considered the most important legislation in the fight against HIV and AIDS, was the expansion of Medicaid coverage to include a number of individuals living below the poverty line and childless adults $[15,16]$. This change expanded access to care for PLWH particularly for those who never had access before [16]. In Indiana, the Medicaid expansion came in the form of an amendment to the Healthy Indiana Plan, now known as HIP 2•0, which was effective February 1, 2015 [17].

With the new healthcare plans in place and their clients enrolled, AMAA observed PLWH still had gaps in care. AMAA recognized that while the expansion of insurance meant more access, it did not guarantee an increase in utilization. Further, they recognized that the gaps in care implied their clients' lack of understanding about the importance of continuity of care as a way to improve their health status but also as a way to eliminate further transmission of HIV. Understanding that barriers to HIV care still exist even after the client had been enrolled, AMAA wanted these barriers identified. Thus, the purpose of this phenomenological study was to qualitatively examine the issue of continuity of care for PLWH after the implementation of the ACA and HIP $2 \cdot 0$ in Northern Indiana.

\section{Methods}

\section{Study Design}

The primary goal of a phenomenological study is to understand the meaning and essence of the lived experiences of a group of people who have experienced a particular phenomenon in a given context while maintaining the principles of scientific rigor [18]. This type of study does not seek to find causes or explanations for the phenomenon but rather seeks a deeper level of understanding of the collective lived experiences [18].

Interview questions were open ended and designed to be exploratory in nature so as to not lead study participants [18,19]. The interview consisted of a few broad, conversation-starter questions to establish the purpose of the interview. This process generated rich data that revealed the participant's thoughts, feelings, and beliefs about his/her lived experiences as a PLWH seeking treatment and care. Follow up questions allowed the researcher to clarify and confirm their understanding of the participants' lived experiences [20].

The individual face-to-face interviews were performed in a location convenient to the client and were audio recorded using digital recording technology. Informed consent was obtained from all study participants. The audiotapes were transcribed verbatim. Transcripts, tapes, and all other data related materials were securely stored.

\section{Study Sample}

Phenomenological studies are generally conducted with small sample sizes (three to ten) because the nature of the task demands extensive study of each participant, "allowing the subjects to speak for themselves and to reveal the logic of their experience as lived" [21]. A purposive sampling strategy was employed which is a non-probability is sampling technique where participants are chosen because of their particular experience and knowledge on a subject the researcher needs to gather information [22]. Study participants were recruited with the assistance of AMAA staff. Participants were chosen based on the following inclusion criteria: (a) PLWH, (b) 18 years or older, (c) individual either had or were eligible for health insurance, and (d) individual experienced a gap in care; period of at least six months after the implementation of ACA and HIP 2.0 where PLWH did not see health care professionals such as an Infectious Disease Specialists and Primary Care doctor or receiving other comprehensive health care services. The study sample consisted of six participants.

\section{Data Analysis}

Inductive data analysis was used to process the raw data; to detect patterns and themes and to generate general conclusions. Inductive data analysis is described as bringing salient pieces of data together to create a 
meaningful whole [23]. The steps for analysis were adapted from Creswell, Saldana, and Hatch, which included: (a) close-reading to gain an overall sense of the information, (b) utilizing descriptive coding to summarize the key concept of an excerpt, (c) creating a summary sheet for each participant based on the descriptive codes, and (d) using an iterative approach to generate themes, identify relationships, find explanations, and generate theories from the coding on the participants summary sheets [23-25]. The researchers engaged in these steps independently and then came together to engage in several discussions about their findings. These additional steps included: (a) sharing codes and patterns identified for each participant, (b) engaging in discourse to connect their findings, (c) identifying the major themes of the data based on codes, patterns and discussion, and finally, (d) interpreting the larger meaning of the data. All field notes, researcher notes, and discussion notes have been maintained for transparency.

\section{Results}

The purpose of the study was to explore the lived experiences of PLWH with insurance who were experiencing gaps in care, which was defined as a period of at least six months after the implementation of ACA and HIP 2.0 where PLWH did not seeing health care professionals such as an Infectious Disease Specialists and Primary Care doctor or receiving other comprehensive health care services. The analysis determined that participants experienced a minimization of traditional barriers to care but experienced two new barriers to care: 1) complexity of health insurance, and 2) recognition of gap in care which were not previously identified in the literature.

\section{Participant Demographics and Health Status}

Table 1 lists the demographics, education level, and employment status of the six study participants was recorded.

\begin{tabular}{|c|c|c|c|c|c|c|c|}
\hline Patient* & Age & Gender & Race & $\begin{array}{c}\text { Marital } \\
\text { Status }\end{array}$ & Education Level & Employed & $\begin{array}{c}\text { Year of HIV } \\
\text { Diagnosis }\end{array}$ \\
\hline C & 45 & M & White & Single & High School Grad & Yes & 2006 \\
\hline D & 34 & F & Black & Single & College Grad & Yes & 2002 \\
\hline E & 51 & M & White & Single & High School Grad & No & 2011 \\
\hline F & 38 & F & Black & Single & Did not graduate High School & No & 2001 \\
\hline G & 25 & M & White & Single & High School Grad & Yes & 2011 \\
\hline H & 42 & M & Black & Single & High School Grad & No & 1993 \\
\hline
\end{tabular}

Table 1: Participant demographics.

* Client A served as a pilot and Client B did not satisfy all the inclusion criteria.

The participants' median age was 40 years; four (66\%) were male and two $(33 \%)$ were female. The participants' education levels were as follows: one (16\%) did not graduate high school, four (66\%) graduated high school and one $(16 \%)$ graduated college. Half of the cohort was employed. The median length of diagnosis was 11 years.
The CD4 counts and viral loads of the study participants, which are health indicators for PLWH, can be found in Table 2. Five (83\%) of the six participants had CD4 counts in the healthy range, however, only one $(17 \%)$ of the six participants had an undetectable viral load. 


\begin{tabular}{|c|c|c|}
\hline Patient & CD4( $\mathbf{m m}^{\mathbf{3}} \mathbf{)} /$ Year & Viral Load(ml)/Year \\
\hline C & 710 in 2015 & $20^{* *}$ in 2013 \\
\hline D & 810 in 2015 & 2050 in 2015 \\
\hline E & 669 in 2016 & 102 in 2016 \\
\hline F & 673 in 2015 & 5170 in 2015 \\
\hline G & 788 in 2015 & 199 in 2015 \\
\hline H & 331 in 2015 & 46,900 in 2015 \\
\hline
\end{tabular}

Table 2: Health status of participants.

* CD4 count considered healthy: $500-1200 \mathrm{~mm}^{3}$

**Viral load considered undetectable: 40 - $75 \mathrm{ml}$ copies

\section{Minimizing Traditional Barriers}

While all participants acknowledged and shared their experiences with traditional barriers to care like transportation, convenience, and stigma, the participants explained that by working with AMAA, they overcame these traditional barriers. "Since ... I've had a relationship with them (AMAA) they've been a world of help" (Client E, interview, September 7, 2016). Although stigma is regarded as a barrier to care, none of the participants described it as such. In fact, one client claimed the topic of HIV has become less taboo. "It's not, you know, a big deal to talk about, like such a shock or stigma that you mention things like that" (Client D, interview, August 19, 2016). All of the study participants spoke about the assistance they received with scheduling and transportation to appointments. Client C credited her care coordinator saying, "Yeah, yeah. She gets the appointments, makes sure the appointments are there... Least I have a worker, someone, a counsellor, that's there because I have no way of getting around, other than by foot" (Client C, interview, July 28, 2016).

\section{Health Insurance Complexity}

The complexities of obtaining, maintaining, and navigating health insurance were discussed in every interview, but, again, the participants did not view these complexities as a barrier to consistent care. However, the data analysis identified it as such because each comment about insurance was accompanied with a comment about the lack of access to a physician or lack of access to medications. For example, Client D reported "I'll say within the last year, healthcare has been more accessible" even though she added "Like right now I don't have a primary care doctor" (Client D, Interview, August 19, 2016). Without access to a primary care doctor Client $D$ is not receiving all the medical care she needs to remain healthy.
Client $\mathrm{C}$ talked about how at one time "I pay nothing for my insurance" (Client C, interview, July 28, 2016) but then something changed without his knowledge and "Just the last time I was there I went for blood work and I just got a bill ... and it's like why am I getting a bill? This is supposed to be $100 \%$ covered" (Client C, interview, July 28, 2016). While Client $\mathrm{C}$ believes he should not have to pay, he gets bills for different procedures and does not understand why. This new form of coverage is foreign to Client $\mathrm{C}$, and unsurprisingly, confusing.

Client D talked about the "repetition" and "absurdity" of the process of obtaining health insurance (Client D, interview, August 19, 2016). She said, "I'm telling all these people all my business, I'm signing a hundred papers and at the end of the day I'm told, oh, you don't qualify, you don't fall below the poverty rate" (Client D, interview, August 19, 2016). Client $G$ talked about a blog he maintains and described ongoing blog discussions about health insurance for PLWH. "Some of the main problems they've been having are they have to reapply again or 'my insurance doesn't cover everything,' 'it only covers this' and 'my insurance covers this but not that.' Um, it's just a back and forth thing and it's frustrating for people" (Client G, interview, November 10, 2016). Client H described his understanding of the insurance information like this: "It was overwhelming cuz it was a lot and I didn't, I didn't clearly understand every single thing" (Client $\mathrm{H}$, interview December 1, 2016). Without being able to understand the details of their insurance plans, the participants have difficulty fully utilizing their coverage and receiving consistent treatment and care.

\section{Recognizing Gap in Care}

Perhaps the most significant finding of this study was the understanding of the phrase gap in care which contributed to a new barrier experienced by participants. 
The interviews revealed a disconnect between the health care seeking behaviour of the participants and the expectations of AMAA. Per AMAA, all participants had not accessed care for more than six months, but none of the study participants recognized their inconsistency in HIV care utilization as a gap. The participants reported feeling fine and did not see the necessity in returning to the doctor as soon as recommended. "Um, because my viral load and CD4 count were the same as when I was tested [at initial diagnosis]. So I said ok... I didn't continuously go to the doctor" explained Client G (Client G, interview, November 10, 2016). Client $\mathrm{H}$ reported, "I was doing so well and at that time when I moved I just didn't take the initiative to get a doctor... since I was doing so well I thought I'm ok... I wasn't sick. I wasn't having any complications. I wasn't losing weight or anything of that nature. So to me I thought I'm doing everything they said to do" (Client $\mathrm{H}$, interview December 1, 2016). All of the participants seem to lack an understanding of a gap in care and the importance of continuity of care. As a result, participants do not fully comprehend the effects inconsistent care has on their health status.

\section{Discussion}

With the implementation of ACA and HIP 2.0, PLWH were expected to increase their HIV care utilization since insurance status has been shown to be one of the strongest factors associated with HIV care utilization..$^{13}$ Conversely, the data in this study demonstrates that sole provision of insurance does not guarantee utilization. Although more than $90 \%$ of AMAA's active clients (including the six study participants) have now been enrolled in health insurance, many clients still do not consistently access care. While this study confirmed AMAA's success to minimize traditional barriers which have been previously identified in the literature, the complexity of health insurance compounded with a lack of recognition of gap in care has unintentionally led to inconsistent care and low health status [5-12].

Indiana's significant changes in health care for PLWH since ACA and HIP $2 \cdot 0$ have been unnecessarily complex, and these health insurance complexities became a barrier to care themselves. [17] These six study participants have been thrust into a difficult position because not only do they have limited experience navigating the health insurance environment, but the analysis shows they also have low health literacy, which is defined as "the degree to which an individual has the capacity to obtain, communicate, process, and understand basic health information and services to make appropriate health decisions" in the Patient Protection and Affordable Care Act of 2010, Title V [26]. There is an assumption that once a person obtains health insurance the person will understand what insurance is and how to use it. However, if an individual's health literacy is low, the individual will have difficulty navigating the healthcare system; filling out complex forms; locating providers and services; sharing appropriate information with their providers; and engaging in self-care and chronic-disease management [27]. In order to remove the barrier of a complex insurance system, study participants need to improve their health literacy and develop a greater degree of selfmanagement which is particularly important as HIV begins to be treated as a chronic disease [28].

The second new barrier to emerge, recognition of a gap in care, was perhaps unexpected. The care coordination community assumes continuum of care for PLWH is understood, but participants did not view their inconsistent HIV treatment and care as problematic as long as they were 'feeling fine.' Nonetheless these participants were not 'fine' as only one participant $(16.7 \%)$ had a viral load in the undetectable range meaning the remaining participants $(83.3 \%)$ are still at risk of transmitting HIV. This viral suppression rate is even lower than the rate for the entire state of Indiana where only $36.7 \%(3,943)$ PLWH had viral suppression [29-31]. This notion of 'feeling fine' and not recognizing their own gap in care demonstrates low health literacy among participants exists.

\section{Conclusion}

As a result of this study, we were able to examine the issue of continuity of care for PLWH after the implementation of the ACA and HIP 2.0 in Northern Indiana. We demonstrated that sole provision of insurance does not guarantee usage and modern barriers prevent PLWH from utilizing HIV care. The system of care for PLWH has evolved tremendously during the past three decades. Initially, HIV was considered an acute illness often resulting in death. As biomedicine has evolved so too has the life expectancy of a PLWH. The findings of this phenomenological study suggests as the system of treatment shifts toward a chronic disease model that care coordination should follow suit in order to reduce barriers and improve access to care. In light of the recent implementation of the ACA and HIP $2 \cdot 0$, improving health literacy should become a priority for care coordination which could include incorporating intentional education regarding navigation of the health insurance system, viral load suppression, and the understanding and importance 
of continuity of care. This strategy may empower and engage study participants to access HIV care more consistently and effectively, thus minimizing barriers, improving self-management and health status, and preventing HIV transmission.

\section{Limitations}

Persons living with HIV in the local community who were not affiliated with AMAA and experienced a gap in care were not interviewed. Additionally, difficulty in scheduling interviews added to the limitations of this study. Many AMAA clients met the study criteria, however, they were difficult to contact or schedule given disruptions in cell phone services, last minute interview cancellations, or no-shows.

\section{Acknowledgements}

The authors gratefully acknowledge AMAA, especially L.H. and B.A. for their advice, guidance, and assistance.

\section{References}

1. Centers for Disease Control and Prevention (2015) Indiana - 2015 State Health Profile. Atlanta, GA: US Department of Health and Human Services.

2. Centers for Disease Control and Prevention (2016) HIV/AIDS HIV Basics. Atlanta, GA: US Department of Health and Human Services.

3. World Health Organization (2015) Guideline on when to start antiretroviral therapy and on pre-exposure prophylaxis for HIV. Geneva: World Health Organization.

4. AIDS Ministries/AIDS Assist of North Indiana Inc (2015) Annual Report 2014. South Bend, Indiana.

5. Cavaleri MA, Kalogerogiannis K, McKay MM, Vitale L, Levi E, et al. (2010) Barriers to HIV care: An exploration of the complexities that influence engagement in and utilization of treatment. Soc Work Health Care 49(10): 934-945.

6. Cunningham WE, Hays RD, Williams KW, Beck KC, Dixon WJ, et al. (1995) Access to medical care and health-related quality of life for low-income persons with symptomatic human immunodeficiency virus. Med Care 33(7): 739-754.
7. Kempf MC, McLeod J, Boehme AK, Walcott MW, Wright L, et al. (2010) A qualitative study of the barriers and facilitators to retention-in-care among HIV-positive women in the rural south eastern United States: Implications for targeted interventions. AIDS Patient Care STDS 24(8): 515-520.

8. Marx R, Hirozawa A, Soskolne V, Liu Y, Katz MH (2001) Barriers to getting needed services for Ryan White CARE clients. AIDS Care 13(2): 233-242.

9. Sagrestano LM, Clay J, Finerman R, Gooch J, Rapino M (2014) Transportation vulnerability as a barrier to service utilization for HIV-positive individuals. AIDS Care 26(3): 314-319.

10. Toth M, Messer L, Quinlivan E (2013) Barriers to HIV care for women of color living in the south eastern US are associated with physical symptoms, social environment, and self-determination. AIDS Patient Care STDS 27(11): 613-620.

11. Williams B, Amico K, Konkle-Parker D (2011) Qualitative assessment of barriers and facilitators to HIV treatment. J Assoc Nurses AIDS Care 22(4): 307312.

12. Wohl AR, Carlos JA, Tejero J, Dierst-Davies R, Daar ES, et al. (2011) Barriers and unmet need for supportive services for HIV patients in care in Los Angeles County, California. AIDS Patient Care STDS 25(9): 525-532.

13. Kalichman SS, Catz S, Ramachandran B (1999) Barriers to HIV/AIDS treatment and treatment adherence among African-American adults with disadvantaged education. J Nat Med Assoc 91(8): 439446.

14. Uphold CR, Mkanta WN (2005) Review: Use of health care services among persons living with HIV infection: State of the science and future directions. AIDS Patient Care STDS 19(8): 473-85.

15. AIDS.gov (2016) The Affordable Care Act and HIV/AIDS.

16. Kates J, Dawson L, Undem T, Perry K (2014) Health Insurance Coverage for People with HIV Under the Affordable Care Act: Experiences in Five States. The Henry J. Kaiser Family Foundation. 
17. The Henry J (2016) Kaiser Family Foundation, Medicaid Expansion in Indiana. The Henry J Kaiser Family Foundation. Menlo Park, CA: The Henry J. Kaiser Family Foundation.

18. Moustsakas C (1994) Phenomenological Research Methods. CA: Sage Publications.

19. Seidman I (2013) Interviewing as qualitative research: A guide for researchers in education and the social sciences. NY: Teachers College Press, Columbia University.

20. Miner-Romanoff K (2012) Interpretive and Critical Phenomenological Crime Studies: A Model Design. Qual Rep 17(27): 1-32.

21. Dukes S (1984) Phenomenological Methodology in the Human Sciences. J Relig Health 23(3): 197-203.

22. Merriam S (2009) Qualitative Research: A Guide to Design and Implementation. CA: Jossey-Bass.

23. Hatch A (2002) Doing qualitative research in education settings. NY: State University of New York Press.

24. Creswell J (2007) Qualitative inquiry and research design: Choosing among five approaches. CA: Sage Publications.
25. Saldana J (2009) The coding manual for qualitative researchers. CA: Sage Publications.

26. Centers for Disease Control and Prevention Health Literacy (2015) Atlanta, GA: US Department of Health and Human Services.

27. Office of Disease Prevention and Health Promotion (2016) Quick Guide to Health Literacy. Rockville, MD: US Department of Health and Human Services.

28. Deeks S, Lewin SR, Havlir DV (2013) The ends of AIDS: HIV Infection as a chronic disease. The Lancet 382(9903): 1525-1533.

29. Centers for Disease Control and Prevention (2015) State HIV Prevention Progress Report, 2010-2013. Atlanta, Georgia: US Department of Health and Human Services.

30. Indiana State Department of Health (2013) 2013 HIV/AIDS Epidemiologic Profile, Indiana. Indiana: Indiana State Department of Health.

31. Centers for Disease Control and Prevention (2016) Learn About Health Literacy. Atlanta: CDC. 